\title{
Análisis comparativo del grado de desarrollo de la coordinación motriz en niños y niñas de educación preescolar
}

\author{
Comparative analysis of the degree of motor development in kindergarten \\ boys and girls \\ Paulina Yésica Ochoa-Martínez ${ }^{1}$, Javier Arturo Hall-López ${ }^{1}$, Daniel Alejandro Piña Díaz ${ }^{1}$, \\ Edgar Ismael Alarcón Meza ${ }^{1}$, Uriel Zúñiga Galaviz ${ }^{2,3}$ \\ 1 Facultad de Deportes, Universidad Autónoma de Baja California (UABC), México. \\ 2 Instituto de Ciencias Biomédicas de la Universidad Autónoma de Ciudad Juárez (UACJ), México. \\ 3 Facultad de Ciencias de la Cultura Física, Universidad Autónoma de Chihuahua (UACH), México.
}

CORRESPONDENCIA:

Javier Arturo Hall-López

javierhall@uabc.edu.mx

Recepción: junio 2019 • Aceptación: enero 2020
CÓMO CITAR EL ARTÍCULO:

Ochoa-Martínez, P. Y., Hall-López, J. A., Piña-Díaz, D. A., AlarcónMeza, E. I., \& Zúñiga-Galaviz, U. (2020). Análisis comparativo del grado de desarrollo de la coordinación motriz en niños y niñas de educación preescolar. Cultura, Ciencia y Deporte, 15(44), 277-283.

\section{Resumen}

El objetivo de la investigación fue comparar por género la coordinación motriz fina, coordinación motriz gruesa y coordinación motora total de niños y niñas de prescolar. El diseño del estudio fue descriptivo comparativo, con muestreo por conveniencia, participaron 179 niños y niñas de una edad promedio de 4 y 5 años matriculados en jardines de niños para educación preescolar de la ciudad de Mexicali, Baja California, México. Se utilizó como instrumento de evaluación el inventario de desarrollo Battelle para determinar la coordinación motriz fina, coordinación motriz gruesa y coordinación motora total. La igualdad de la varianza se calculó mediante la prueba t Student para muestras independientes, resultando por género menor $a \mathrm{a} \leq 0.05$; la coordinación motriz gruesa (P-Valor=.000), coordinación motriz fina (P-Valor=.002) y la coordinación motora total (P-Valor=.000). La comparación mostró diferencias significativas en diversas capacidades motoras entre niños y niñas, por lo anterior es necesario mejorar las deficiencias motoras por medio de la educación física que permitan un óptimo desarrollo motriz, con actividades igualitarias y mayores oportunidades de participación para las niñas.

Palabras clave: educación física, educación preescolar, desarrollo motor, enseñanza deportiva.

\begin{abstract}
The aim of the study was to compare by gender the fine motor coordination, gross motor coordination and total motor coordination of kindergarten children. The research design was comparative descriptive, with convenience sampling, involving 179 children of an average age of 4 and 5 years enrolled in kindergartens for preschool education in the city of Mexicali, Baja California. Mexico. The Battelle development inventory was used as an evaluation instrument to determine fine motor coordination, gross motor coordination and total motor coordination. The equality of the variance was calculated by the Student $t$ test for independent samples resulting in gender less than $a \leq$ 0.05; gross motor development ( $P$-Value $=.000)$, fine motor development ( $\mathrm{P}$-Value $=.002)$ and general motor development ( $P$-Value=.000). The comparison showed significant differences in motor coordination between boys and girls, therefore it is necessary to improve motor deficiencies through physical education that allow optimal movement development, with equal activities and greater opportunities for participation for girls.
\end{abstract}

Key words: Physical education, kindergarten education, motor development, sports education. 


\section{Introducción}

Dentro de los objetivos de desarrollo sostenible propuestos por la Organización de las Naciones Unidas (ONU) para disminuir la pobreza extrema en sus varias dimensiones se encuentra la igualdad de género, abarcando el ámbito educativo (Pérez Betancourt, \& Betancourt Rodríguez, 2019) y permeando entornos pedagógicos de diferentes niveles (Pastor Gosálbez et al., 2019), como lo es el universitario, donde en el ámbito de la educación física y las ciencias de la actividad física y el deporte se encuentra presente en la formación sobre género (Fernández García, \& Piedra de la Cuadra, 2013, Rodríguez Ojeda, Rodríguez Iglesias, \& Pérez González, 2018). La Organización de las Naciones Unidas para la Educación, la Ciencia y la Cultura (UNESCO) ha recomendado, dentro de las guías para los responsables políticos que laboran en torno a la educación física, que la escuela sea un elemento clave para proporcionar a las niñas información, competencias y confianza necesaria para la práctica de la actividad física y del deporte durante toda la vida (McLennan, \& Thompson, 2015).

Respecto a la actividad física los resultados de la Encuesta Nacional de Salud y Nutrición de Medio Camino, ENSANUT MC 2016, refieren que las niñas realizan menor cantidad de actividad física (12.7\%) respecto a los niños (17.2\%); investigaciones que evalúan la actividad física en la jornada escolar reflejan un patrón similar en cuanto a la cantidad de actividad física, reportando mayor actividad física moderada a vigorosa en niños que en niñas (Hall-López et al., 2017; HallLópez et al., et al., 2018; Hall-López et al., et al., 2019).

De acuerdo con la Organización Mundial de la Salud (OMS), el desarrollo motriz en la primera infancia (etapa que se extiende desde el desarrollo prenatal hasta los ocho años de edad) es crucial para su optimo bienestar, crecimiento y es una influencia clave en el posterior ciclo de vida de un individuo (OMS, 2016); un estudio evaluó la variable de edad motora equivalente en niños de preescolar, utilizando como instrumento de ayuda diagnóstica el inventario de desarrollo Battelle (Newborg et al., 1996) y correlacionan que los niños con una adquisición motora esperada para su edad presentan en mayor medida un igual desarrollo cognitivo y lenguaje acorde a su edad (Campo Ternera, 2010). En México el programa de educación física para preescolar presenta un enfoque didáctico enfocado en el desarrollo de la motricidad, integración de la corporeidad y la creatividad en la acción motriz (SEP, 2017). Al revisar el estado del arte, los factores personales y sociales vinculados a la práctica físico-deportiva desde la perspectiva del género, son multifactoriales (Torre Ramos, 2002;
Vicente-Pedraz, \& Paz Brozas-Polo, 2017). De acuerdo a nuestro conocimiento, ha sido complejo identificar estudios específicos que evalúen la motricidad de acuerdo al género por lo anterior, el presente estudio tiene como objetivo realizar un análisis comparativo por género la coordinación motriz fina, coordinación motriz gruesa y coordinación motora total de niños de segundo y tercero grado de preescolar.

\section{Método}

\section{Participantes}

La presente investigación fue aprobada y registrada en la Coordinación de Posgrado e Investigación de la Universidad Autónoma de Baja California y se llevó acabo entre agosto de 2017 y agosto de 2018 bajo un diseño metodológico transversal comparativo, con muestreo no probabilístico por conveniencia (Thomas, Nelson, \& Silverman, 2015), solicitando anuencia de participación a los directivos y profesores de las instituciones educativas, programando los horarios de evaluación y siguiendo los principios éticos de investigación en seres humanos de la declaración de Helsinki (Puri, Suresh, Gogtay, \& Thatte, 2009). Se evaluó el desempeño motor de los niños y niñas con edad de 4 y 5 años en las instituciones de educación preescolar públicas de la ciudad de Mexicali, siendo 179 las muestras totales, estableciendo como variable fija a dos grupos, el primero formado por 87 individuos del sexo masculino con edad de 4-5 años, y el segundo grupo de 92 individuos del sexo femenino, determinando en cada grupo los valores de tres variables aleatorias: 1 ) Índice del desarrollo de motricidad fina, 2) Índice del desarrollo de motricidad gruesa y 3) Índice del desarrollo de motricidad total, cuyas evaluaciones se detallan a continuación en los procedimientos.

\section{Instrumentos}

La variable que determina la edad motora equivalente en meses de los sujetos participantes se obtuvo utilizando el Inventario de Desarrollo Battelle, (Newborg, Stock, \& Wnek, 1996), que se trata de una batería para evaluar las habilidades fundamentales del desarrollo en niños comprendidos entre el nacimiento y los ocho años; su aplicación es individual y está tipificada. Es un inventario objetivo que, durante sus procedimientos para la obtención de datos, utiliza la observación y usa un examen estructurado. Los ítems se presentan en un formato normalizado que especifica la conducta que se va a evaluar, los materiales necesarios, los 
procedimientos de administración y los criterios para puntuar la respuesta. Su aplicación está compuesta por 341 ítems para el total del rango de edades al que está destinada. Examina las siguientes áreas del desarrollo:

- Personal/Social. Evalúa las capacidades y características que permiten al niño establecer interacciones sociales significativas.

- Adaptativa. Se centra en la capacidad del niño para utilizar la información y las habilidades evaluadas en las otras áreas.

- Motora (Motricidad Gruesa y Motricidad Fina). Se encuentra en la evaluación del desarrollo motor grueso y la capacidad del niño para usar y controlar los músculos del cuerpo.

- Comunicación (Receptiva y Expresiva). En esta área se evalúa la recepción y expresión de la información y los pensamientos e ideas por medios verbales y no verbales.

Para los fines de la investigación se enfatiza determinar la puntuación para su edad del área de desarrollo motor, la cual consta de 5 subáreas; son un total de 82 ítems evaluados.

1. Control Muscular: evalúa el desarrollo motor grueso y la capacidad del niño para establecer y mantener el control, principalmente sobre los músculos que utiliza para sentarse, estar de pie, pasar objetos de una mano a otra y realizar tareas semejantes.

2. Coordinación Corporal: evalúa aspectos del desarrollo motor grueso: la capacidad del niño para utilizar su sistema muscular y para establecer un control y una coordinación corporal cada vez mayores (por ejemplo, cambiar la posición del cuerpo, rodar en el suelo, dar patadas, tirar y recoger objetos, dar saltos, hacer flexiones y realizar saltos de longitud).

3. Locomoción: evalúa aspectos del desarrollo motor grueso: la capacidad del niño de utilizar los sistemas de musculatura de forma integrada con el fin de trasladarse de un sitio a otro (por ejemplo, arrastrarse, gatear, andar, correr, saltar o subir y bajar escaleras).

4. Motricidad Fina: evalúa el desarrollo del control y coordinación muscular del niño, especialmente de la musculatura fina de brazos y manos que permite llevar a cabo tareas cada vez más complejas como tomar y soltar objetos, abrir y cerrar puertas y cajones, ensartar cuentas, pasar páginas, cortar, doblar papel y utilizar el lápiz correctamente.

5. Motricidad Perceptiva: evalúa aspectos del desarrollo motor fino: la capacidad del niño para integrar la coordinación muscular y las habilidades perceptivas en actividades concretas, como formar torres, colocar anillas en un soporte, copiar círculos, cuadrados, dibujar y escribir.

\section{Procedimientos}

La prueba se realizó en un lapso de entre una hora a una hora y treinta minutos, realizando un procedimiento dividido en tres pasos: uno es obtener información de los padres, tutores o el profesor, el segundo se llevó a cabo la aplicación del examen estructurado y, en el tercero, análisis observacionales de la conducta que se llevó a cabo en el ambiente del niño.

Antes de comenzar con la prueba fue muy importante tener en cuenta los siguientes puntos: que se conozca y domine la metodología para aplicar el inventario de desarrollo Batelle, la seguridad que las condiciones ambientales para llevar a cabo la prueba y la confianza, no solo para el que lo aplica si no para aquel a quien se realiza la prueba.

Para registrar el primer paso se comenzó en el ítem correspondiente a la edad de cada niño o niña. Si al realizar la prueba no consigue los dos puntos en el ítem se le aplicarán todos los elementos de ese nivel y se prosigue retrocediendo un nivel inferior para encontrar el umbral (ya sea por debajo del cual el niño puntuará 2 en todos los elementos), o se continúa con los demás ítems hasta encontrar el techo (por encima del cual todas las puntuaciones serán "0" o de otra forma se puede evaluar tomando 0 como nunca, 1 como a veces y 2 como siempre). Con estos valores se realiza una suma que proporciona una puntuación total cuyo resultado se compara con las tabla de resumen de puntuaciones y perfil del inventario, estableciendo así la edad motora en meses equivalente del niño a partir del control muscular, coordinación corporal, locomoción, motricidad fina y motricidad perceptiva, clasificándose de tres maneras: 1. Alto: por encima de lo esperado para su edad, 2. Normal: de acuerdo a los patrones normativos de la edad y 3. Bajo: por debajo de lo esperado de su edad.

\section{Análisis Estadístico}

El análisis estadístico se realizó mediante el Paquete Estadístico para las Ciencias Sociales (SPSS), versión 23.0 para Windows (IBM Corporation, New York, USA), calculando los valores descriptivos de las variables; y el porcentaje de diferencia $(\Delta \%)$ ([(Media-2 Media-1)/Media-1] x 100) (Vincent, 2012).

De acuerdo a la metodología utilizada en la presente investigación se estableció una prueba de hipótesis alterna y otra nula; para verificar la normalidad de los grupos y homogeneidad de la varianza de los datos se utilizó el test estadístico de Kolmogorov-Smirnov, debido a que fue una cantidad mayor a 30 evaluaciones 
Tabla 1. Estadística descriptiva por género de las variables estudiadas por el inventario de desarrollo Batelle.

\begin{tabular}{|c|c|c|}
\hline \multirow{2}{*}{ Variables } & \multicolumn{2}{|c|}{$M \pm D E$} \\
\hline & NIÑOS $(n=87)$ & NIÑAS $(n=92)$ \\
\hline Edad en meses de desarrollo para subárea motora gruesa. & $74.1 \pm 92$ & $54.3 \pm 8.7$ \\
\hline Edad en meses de desarrollo para subárea motora fina. & $66.8 \pm 8.7$ & $49.2 \pm 7.9$ \\
\hline Edad en meses de desarrollo para área motora total. & $69.9 \pm 7.6$ & $51.8 \pm 8.3$ \\
\hline
\end{tabular}

Nota: Los valores presentados de los sujetos son media y desviación estándar ( \pm ) de la puntuación típica obtenida de los sujetos de estudios mediante las pruebas del inventario de desarrollo Batelle (Newborg et al., 1996).

en las variables fijas que incluyó el desarrollo del área motora en sus tres fraccionadas sub áreas cubriendo el área motriz en su totalidad. En ambas se aprecia un grado de significancia de P-Valor $<0.05$. Como estudio transversal compara como variables fijas dos grupos de niños de distinto sexo, teniendo como variables aleatorias numéricas: 1) desarrollo del área motora fina, 2) desarrollo del área motora gruesa y 3) desarrollo del área motora total. Se utilizó estadística inferencial, la prueba $t$ Student para muestras independientes con la finalidad de calcular la igualdad de la varianza, determinando un nivel de $\alpha \leq 0.05$, es decir un $5 \%$ como porcentaje de error de la prueba estadística. Los resultados estadísticos se procesaron en forma de tabla y figura.

\section{Resultados}

En la investigación se evaluó el desarrollo de las áreas motoras de niños que presentan 4-5 años, siendo estos divididos en dos grupos por género, un grupo de niños y un grupo de niñas con la misma edad. La resultante de media para el área motriz total que presentan las niñas de 48 a 71 meses de desarrollo cronológico es 51.8 (límite inferior 47.7 a 56.3 límite superior) en comparación de la media para el área motriz total que presentan los niños es de 69.9 meses de desarrollo (límite inferior 67.5 a 72.4). La estadística descriptiva sobre las diversas áreas motoras evaluadas mediante el sistema para observar el desarrollo biológico motor de los grupos de niños durante la etapa preescolar que presentan 4 y 5 años cronológicamente (Rango de 48 a 71 meses) se puede observar en la tabla 1 .

De acuerdo a la metodología utilizada la presente investigación se estableció una prueba de hipótesis alterna y otra nula de la siguiente manera:

La hipótesis alterna $\mathrm{H}_{1}$ : existe una diferencia significativa entre las medias del desarrollo motor en la subárea motora gruesa, subárea motora fina y área motora total en niños de 48 a 71 meses de edad cronológica en comparación de las niñas que presentan igual desarrollo cronológico que están matriculados en los jardines de educación preescolar de la ciudad de Mexicali.

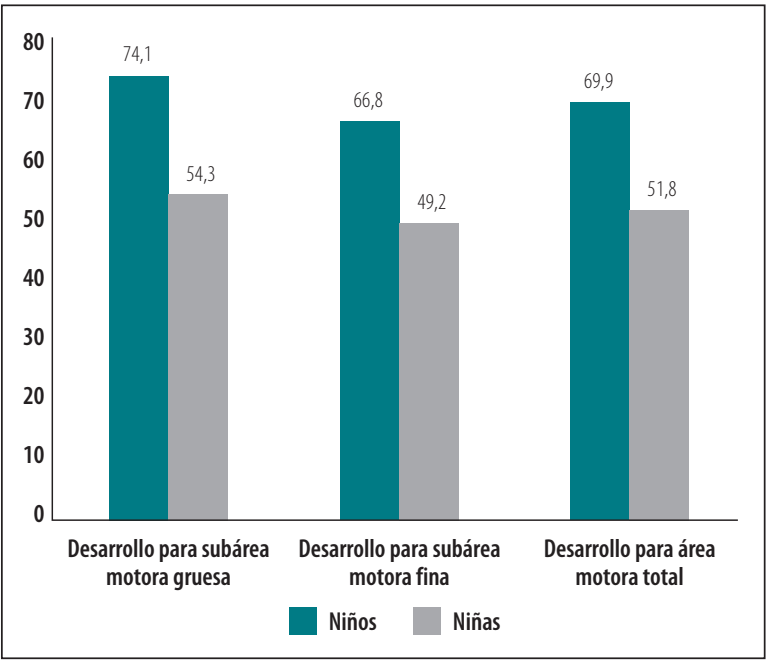

Figura 1. Estadística inferencial por género de las variables estudiadas por el inventario de desarrollo Batelle.

Nota: Cálculo de la varianza de las variables mediante el test t student para muestras independientes ( $a \leq 0.05$ ), encontrando diferencias significativas en el la subárea motora gruesa (.000), con el inventario de desarrollo Battelle ( $n=179)$. (Newborg et al., 1996).

Hipótesis nula, $\mathrm{H}_{0}$ : no existen diferencias entre las medias del desarrollo motor en la subárea motora gruesa, subárea motora fina y área motora total en niños de 48 a 71 meses de edad cronológica en comparación de las niñas que presentan igual desarrollo cronológico que están matriculados en los jardines de educación preescolar de la ciudad de Mexicali.

En el análisis del test estadístico de KolmogorovSmirnov y Shapiro Wilk el valor resulto con una PValor $\geq 0.05$ en el subárea motora gruesa, indicando normalidad de los grupos y la homogeneidad de la varianza de los datos, resultando en la variable fija (.053), subárea motora fina (.008) y para el área motora total (.034) de niños de 48 a 71 meses de edad cronológica que están matriculados en los jardines de educación preescolar de la ciudad de Mexicali. En la figura 1 se muestra la igualdad de la varianza, que se calculó mediante la prueba t Student para muestras independientes, resultando un nivel de significancia de P-Valor menor a $\alpha \leq 0.05$ en la subárea motora gruesa (P-Valor $=.000)$, subárea motora fina $(\mathrm{P}-$ Valor $=.002)$ y el área motora total (P-Valor=.000). 


\section{Discusión}

El resultado principal que en los estudiantes de género masculino presentan de manera significativa un mayor desarrollo motor que el género femenino y de manera descriptiva el porcentaje de diferencia resultó en la coordinación motriz gruesa $(\Delta \% 16.7)$, coordinación motriz fina ( $\Delta \% 10.6)$, y coordinación motora $(\Delta \% 12.2)$. Por otra parte, al comparar por género la clasificación catalogada como baja del desarrollo motor total se observó un mayor porcentaje mayor de niñas $(27.5 \%)$ que en niños (15.5\%) que, de acuerdo a la evaluación, presentan dificultades motrices que requirieren intervención y estimulación en el área de las capacidades coordinativas. Una investigación en estudiantes de preescolar evaluándose con el inventario de desarrollo Battelle se asocia con nuestros resultados, demostrando que un adecuado desarrollo motor se relaciona también con un adecuado desarrollo cognitivo y de lenguaje, lo cual favorece el desarrollo integral de los niños (Campo Ternera, 2011). Una investigación ha demostrado de manera significativa mejor desarrollo motor grueso en los estudiantes de preescolar que cuentan con profesor de educación física (Luna, \& Poblete, 2011; Luarte, Poblete, \& Flores, 2014, Bermudez, et al., 2018). Se afirma que más tiempo dedicado a clases de educación física favorece el desarrollo motor en los estudiantes de preescolar (Jiménez Díaz \& Araya Vargas, 2010). En torno a este tema se han recomendado estrategias didácticas para minimizar la desigualdad (López Pastor, 2012) que favorecen, por medio del movimiento, el desarrollo motriz en estudiantes de prescolar, dentro de las cuales están la actividad física moderada a vigorosa como juego, brindando muchas oportunidades de participar en las acciones motrices independientemente del género (Palma, Pereira, \& Valentini, 2014; García-Puchades, \& ChivaBartoll, 2018), así como el uso de material didáctico que favorezca la igualdad de género (Moya-Mata, et al., 2019). El profesorado juega un papel importante en la conducción directa de los contenidos en los programas educativos evitando estereotipos relacionados al género y dando la importancia de la igualdad de participación de los estudiantes en la clase de educación física (Alvariñas-Villaverde, \& Pazos-González, 2018).

\section{Conclusiones}

El inventario de desarrollo inventario Battelle es metodológicamente de fácil aplicación, económico y proporciona información relacionada a la coordinación motora que puede ayudar al profesor de educación físi- ca a diagnosticar niños con dificultades de movimiento y diseñar actividades educativas enfocadas a cada edad y condición (Sanz López, Guijarro Granados, \& Sánchez Vázquez, 2007; Rizzoli-Córdoba, et al., 2013) y realizar un trabajo multidisciplinario en torno a los niños con desarrollo motor deficiente (Barra Cabello, 2019) y tomar en cuenta otras desigualdades como el contexto educativo, ya que se ha identificado que preescolares que estudian en el sector público (30.4\%) presentan un porcentaje mayor de desarrollo alterado que en el sector privado (14.4\%) (Bedregal et al., 2016), de igual manera que se ha identificado que en preescolares el nivel de desarrollo motor grueso para la edad se encuentra en mejores niveles en población rural que en población urbana (Poblete et al., 2016), así como que la condición socioeconómica, contexto familiar y la actividad física extraescolar son factores importantes a considerar en el desarrollo motor infantil (HerreraMora, 2019; Chiva-Bartoll, \& Estevan, 2019).

De igual forma se ha identificado que niños diagnosticados con sobrepeso y obesidad presentan una desigualdad de desarrollo motor comparado con niños con peso normal (Bucco \& Zubiaur, 2015). El desarrollo motor se ha estudiado en niveles educativos superiores en primaria y secundaria comparando capacidades y habilidades coordinativas, resultando mayor en el género masculino que en el femenino (Cenizo Benjumea, et al., 2016; Torralba, et al., 2016; GuijarroRomero, Mayorga-Vega, \& Viciana, 2019).

Como limitación se establece no contar con conocimiento previo los antecedentes de actividad física de los sujetos de estudio. En el futuro sería importante realizar un número mayor de estudios que clarificaran situaciones causa-efecto respecto a la motricidad de niños de edad preescolar, que sirvieran como referencia en el proceso enseñanza aprendizaje para intervenciones educativas orientadas a la igualdad de género y la mejora en las capacidades coordinativas en esta etapa de la vida. Se debería ampliar la información para los profesores de la educación física y que estos pudieran implementar procesos pedagógicos y curriculares que coadyuvaran desde la primera infancia a mejorar el desarrollo integral, tal como recomienda la Organización Mundial de la Salud (OMS).

\section{Financiación}

Esta investigación fue financiada por el Consejo Nacional de Ciencia y Tecnología (CONACYT) del Padrón Nacional de Posgrados de Calidad (PNPC), aportando fondos a estudiantes para estudiar en la Universidad Autónoma de Baja California, México. 


\section{BIBLIOGRAFÍA}

Alvariñas-Villaverde, M., \& Pazos-González, M. (2018). Estereotipos de género en Educación Física, una revisión centrada en el alumnado. $R e$ vista electrónica de investigación educativa, 20(4), 154-163. https:// dx.doi.org/10.24320/redie.2018.20.4.1840

Barra Cabello, L. (2019). Desafío diagnóstico e importancia del abordaje clínico del trastorno del desarrollo de la coordinación. Archivos argentinos de pediatría, 117(3), 199-204. https://dx.doi.org/10.5546/ aap.2019.199

Bermudez, M., Poblete, F., Pineda, A., Castro, N., \& Inostroza, F. (2018). Nivel de desarrollo motor grueso en preescolares de México sin profesores de educación física. Revista Ciencias de la Actividad Física UCM, 19(1), 1-7. doi: http://doi.org/10.29035/rcaf.19.1.8

Bedregal, P., Hernández, V., Mingo, M. V., Castañón, C., Valenzuela, P., Moore, R., de la Cruz, R., \& Castro, D. (2016). Desigualdades en desarrollo infantil temprano entre prestadores públicos y privados de salud y factores asociados en la Región Metropolitana de Chile. Revista chilena de pediatría, 87(5), 351-358. https://dx.doi.org/10.1016/j. rchipe.2016.02.008

Bucco, L. \& Zubiaur, M. (2015). Análisis del desarrollo motor en escolares brasileños con medidas corporales de obesidad y sobrepeso / Analysis of the Motor Development in Brazilian Schoolchildren with Corporal Measures of Obesity and Overweight. Revista Internacional de Medicina y Ciencias de la Actividad Física y el Deporte, 15(59) 593 611. http://dx.doi.org/10.15366/rimcafd2015.59.012

Camacho-Miñano, M., \& Girela-Rejón, M. (2017). Evaluación de una propuesta formativa sobre género en Educación Física para estudiantes de Ciencias de la Actividad Física y el Deporte. (Evaluation of a proposal for training in gender in Physical Education among students of Physical Activity and Sport Science Studies). Cultura, Ciencia y Deporte, 12(36), 195-202. doi:http://dx.doi.org/10.12800/ccd.v12i36.950

Campo Ternera, L. A. (2010). Importancia del desarrollo motor en relación con los procesos evolutivos del lenguaje y la cognición en niños de 3 a 7 años de la ciudad de Barranquilla (Colombia). Salud Uninorte, 26(1), 65-76.http://www.scielo.org.co/scielo.php?script=sci_ arttext\&pid=S0120-55522010000100008\&lng=en\&tlng=

Cenizo Benjumea, J. M., Ravelo Afonso, J., Morilla Pineda, S., Ramírez Hurtado, J. M. \& Fernández-Truan, J. C. (2016) Diseño y validación de instrumento para evaluar coordinación motriz en primaria / Design and Validation of a Tool to Assess Motor Coordination in Primary. Revista Internacional de Medicina y Ciencias de la Actividad Física y el Deporte, 16(62) 203-219. http://dx.doi.org/10.15366/rimcafd2016.62.002

Chiva-Bartoll, O. \& Estevan, I. (2019). El sexo, el contexto familiar y la actividad física extraescolar como factores asociados a la coordinación motriz en la niñez. Un estudio piloto. RICYDE. Revista internacional de ciencias del deporte, 56(15), 154-170. https://doi.org/10.5232/ricyde2019.05603

Encuesta Nacional de Salud y Nutrición Medio Camino 2016 ENSANUT MC 2016 data revisited. (n.d.). Retrieved feb 17, 2019, from Instituto Nacional de Salud Pública; 2017. Website, http://ensanut.insp.mx/

Fernández García, E., \& Piedra de la Cuadra, J. (2013). Efecto de una formación coeducativa sobre las actitudes hacia la igualdad en el futuro profesorado de Educación Primaria. (Effects of a Coeducative Training on Attitudes Towards Equality in Future Primary Education Teachers).. Cultura, Ciencia y Deporte, 5(15), 151-158. doi:http://dx.doi. org/10.12800/ccd.v5i15.106

García-Puchades, W., \& Chiva-Bartoll, Ó. (2018). El juego como proceso de subjetivación y su justificación en el currículum de educación física. (The play as a process of subjectivation and its justification in the curriculum of Physical Education). Cultura, Ciencia y Deporte, 13(38), 147-156. doi:http://dx.doi.org/10.12800/ccd.v13i38.1070

Guijarro-Romero, S., Mayorga-Vega, D., \& Viciana, J. (2019). Influencia del género sobre la habilidad táctica y aspectos motivacionales en deportes de invasión en Educación Física. (Influence of gender on the tactical skill and motivational aspects in invasion sports in Physical Education).. Cultura, Ciencia y Deporte, 14(41), 93-105. doi:http:// dx.doi.org/10.12800/ccd.v14i41.1269
Hall-López, J. A., Ochoa-Martínez, P. Y., Meza, F., Sánchez, R., \& SáenzLópez, P. (2019). Comparación de la actividad física por género y grasa corporal en escolares mexicanos. Revista Iberoamericana de Ciencias de la Actividad Física y el Deporte, 8(1),1-14. doi:http://dx.doi. org/10.24310/riccafd.2019.v8i1.5763.

Hall-López, J. A., Ochoa-Martínez, P. J., Macías, R. A., Zuñiga, R., \& Sáenz-López, P. (2018). Actividad física moderada a vigorosa en educación física y recreo en estudiantes de primaria y secundaria de la frontera México-USA. Sportis: Revista Técnico-Científica del Deporte Escolar, Educación Física y Psicomotricidad, 4(3), 426-442. http://revistas. udc.es/index.php/SPORTIS/article/view/sportis.2018.4.3.3175

Hall-López, J. A., Ochoa-Martínez, P. Y., Meza Correa, F., López Campos, C. E. \& Sáenz-López Buñuel, P. (2017). Actividad física y percepción de esfuerzo por género en educación física y recreo de escolares mexicanos. Educación Física y Deporte, 36(2), 1-9. http://aprendeenlinea. udea.edu.co/revistas/index.php/educacionfisicaydeporte/issue/ view/3390/showToc

Herrera-Mora, D. B., Munar-Torres, Y. E., Molina-Achury, N. J., \& Robayo-Torres, A. L. (2019). Desarrollo infantil y condición socioeconómica. Artículo de revisión. Revista de la Facultad de Medicina, 67(1), 145-152. https://dx.doi.org/10.15446/revfacmed.v67n1.66645

Jiménez Díaz, J., \& Araya Vargas, G. (2010). Más minutos de educación física en preescolares favorecen el desarrollo motor. Pensar en movimiento, 8(1), 1-8. doi:10.15517/PENSARMOV.V8I1.442

Luarte, R. C., Poblete, V. F., \& Flores, R. C. (2014). Nivel de desarrollo motor grueso en preescolares sin intervención de profesores de educación física, Concepción, Chile. Ciencias De La Actividad Física UCM, 15(1), 7-16. http://revistacaf.ucm.cl/article/view/36

Luna, P., \& Poblete, F. (2011). Desarrollo motor en escolares sin intervención de profesionales de la educación física del nb1 del microcentro "amanecer" de la comuna de nacimiento (región del Biobío, Chile). Revista Horizonte Ciencias De La Actividad Física, 2(2), 25-35. http://www. revistahorizonte.ulagos.cl/index.php/horizonte/article/view/27

López Pastor, V. M. (2012). Didáctica de la educación física, desigualdad y transformación social. Estudios pedagógicos (Valdivia), 38: 155176. https://dx.doi.org/10.4067/S0718-07052012000400009

McLennan, N. \& Thompson, J.: Educación física de calidad (efc): guía para los responsables políticos. Organización de las Naciones Unidas para la Educación, la Ciencia y la Cultura UNESCO. 2015. I.S.B.N. 978-923-300012-4. https://unesdoc.unesco.org/ark:/48223/pf0000231340

Moya-Mata, I., Ruiz-Sanchis, L., Martín Sanchis, J., \& Ros Ros, C. (2019). Estereotipos de género en las imágenes que representan las actividades en el medio natural en los libros de Educación Física de Primaria. (Gender stereotypes in the pictures that represent the activities in the wild in the books of Physical Education Primary). Cultura, Ciencia y Deporte, 14(40), 15-23. doi:http://dx.doi.org/10.12800/ccd.v14i40.1222

Newborg, J., Stock, J, R., \& Wnek, L. (1996). Inventario de Desarrollo Battelle, Madrid, Publicaciones de Psicología aplicada TEA.

Palma, M., Pereira, B., \& Valentini, N. (2014). Guided play and free play in an enriched environment: Impact on motor development. Motriz: Revista de Educação Física, 20(2), 177-185. https://dx.doi.org/10.1590/ S1980-65742014000200007

Pastor Gosálbez, I., Acosta Sarmiento, A., Torres Coronas, T., \& Calvo Merino, M. (2019). Los planes de igualdad en las universidades españolas. Situación actual y retos de futuro. Educación XX1, 23(3), 1-28. doi:https://doi.org/10.5944/educxx1.23873

Pérez Betancourt, A., \& Betancourt Rodríguez, M. Z. (2019). El enfoque de género desde la formación docente y su relación con la ciencia, la tecnología y la sociedad. Revista Boletín Redipe, 8(3), 200-208. https:// revista.redipe.org/index.php/1/article/view/713

Poblete, F., Guerra, R., Toro, P., \& Cruzat, E. (2016). Desarrollo motor grueso en escolares de zona urbana y rural. Revista Horizonte Ciencias De La Actividad Física, 7(1), 59-66. http://revistahorizonte.ulagos.cl/ index.php/horizonte/article/view/73

Puri, K. S., Suresh, K. R., Gogtay, N. J., y Thatte, U. M. (2009). Declaration of Helsinki implications for stakeholders in research. Journal of Postgraduate Medicine, 55(2), 131-134. doi:10.4103/0022-3859.52846 
Rizzoli-Córdoba, A., Schnaas-Arrieta, L., Liendo-Vallejos, S., Buenrostro-Márquez, G., Romo-Pardo, B., Carreón-García, J., Valadez-Correa, E., Scherer-Ibarra, P., López-Aranda, V., Lira-Guerra, S., RoblesAnaya, R., Pizarro-Castellanos, M., Briones-Sandoval, A., Lia-Pirola, M., \& Muñoz-Hernández, O. (2013). Validación de un instrumento para la detección oportuna de problemas de desarrollo en menores de 5 años en México. Boletín médico del Hospital Infantil de México, 70(3), 195-208. http://www.scielo.org.mx/scielo.php?script=sci_ arttext\&pid=S1665-11462013000300003\&lng=es\&tlng=es.

Rodríguez Ojeda, M., Rodríguez Iglesias, A., \& Pérez González, E. M. (2018). Comunicación y educación de género en entornos pedagógicos. ALCANCE Revista Cubana de Información y Comunicación, 7(18), 7892. http://www.alcance.uh.cu/index.php/RCIC/article/view/159/154

Sanz López, Y., Guijarro Granados, T., \& Sánchez Vázquez, V. Inventario de Desarrollo Battelle como instrumento de ayuda diagnóstica en el autismo. Revista de la Asociación Española de Neuropsiquiatría, 27(2), 3145. http://scielo.isciii.es/scielo.php?script=sci_arttext\&pid=S021157352007000200004\&lng=es.

Secretaria de Educación Pública (SEP) Aprendizajes Clave para la Educación Integral. Educación Física. Educación Básica. Plan y Programas de Estudio y sugerencias de evaluación. Primera edición 2017, Ciudad de México. ISBN: 978-607-97644-4-9. Disponible también en la website: https://www.aprendizajesclave.sep.gob.mx/

Thomas, J.R., Nelson, J.K., \& Silverman, S.J. Research Methods in Physical Activity (7th. Ed.). Human Kinetics. 2015. Champaign, Ilinois: Human Kinetics. ISBN: 9781450470445. PUBLICADO: http://www. humankinetics.com/products/all-products/research-methods-inphysical-activity-7th-edition

Torralba, M. A.,Vieira, M. B., Lleixà, T., \& Gorla, J. I. (2016). Evaluación de la coordinación motora en educación primaria de Barcelona y provincia / Assessment of Motor Coordination in Primary Education of Barcelona and Province. Revista Internacional de Medicina y Ciencias de la Actividad Física y el Deporte, 16(62), 355-371. doi: http://dx.doi. org/10.15366/rimcafd2016.62.011

Torre Ramos, E. (2002). Factores personales y sociales vinculados a la práctica físico-deportiva desde la perspectiva del género. Apunts. Educación física y deportes, 4(70), 83-89. https://www.raco.cat/index.php/ ApuntsEFD/article/view/305889

Vicente-Pedraz, M., \& Paz Brozas-Polo, M. (2017). Sexo y género en la contienda identitaria del deporte. Propuesta de un debate sobre la competición deportiva multigénero. (Sex and gender in the contest of identity in sport. A proposal for a debate on multi-gender sports competition). Cultura, Ciencia y Deporte, 12(35), 101-110. doi:http:// dx.doi.org/10.12800/ccd.v12i35.881

Vincent WJ. Statistics in kinesiology (4nd Ed.). Champaign, Ilinois: Human Kinetics; 2012.ISBN-13: 978-1450402545, ISBN10: 1450402542 http://www.humankinetics.com/products/all-products/statistics-in-kinesiology-4th-edition

World health Organization WHO data revisited. (n.d.). Retrieved january 16, 2016, from el Desarrollo de la primera infancia: un potente ecualizador; http://www.who.int/maternal_child_adolescent/documents/ecd_final_m30/es 